and interested educators.

Project STARWALK is currently in use by elementary schools in fifteen states. Some of the schools use the services of the staff at fixed-base planetariums for conducting the student planetarium lessons. Other schools, in more rural areas, have purchased a portable planetarium system for use with STARWALK lessons. In these situations, the teachers have been trained to teach the planetarium lessons as well as the classroom lessons.

\title{
Teaching Astronomy at School in a Planetarium
}

M.F. Duval and D. Bardin

Observatoire de Marseille, 2 Place Le Verrier, 13248 Marseille Cedex 4, France

\section{Introduction}

In some countries, such as the U.S.A., there are quite a lot of major planetariums and an enormous number of small fixed-operating planetariums. In France, the situation is not as good. There are only three planetariums with domes in excess of 15 meters across, six with diameters between six and nine meters, and ten transportable ones. For these last - five EX3s, three Starlabs and two of personal design - the inflatable domes are between four and five meters in diameter, providing a dedicated astronomy teaching facility significantly large to accommodate a typical class.

The principal advantages of these transportable planetariums are:

- easy transportation

- installation inside the school

- quickly erected in less than 20 square meters of space

- capacity of six to eight sessions per day

- low cost: less than $\$ 30$ per class and often free

- good contact between the instructor and the students, who sit on the ground or on small stools in a confined space.

Balancing these great advantages, the main deficiency is the limited program: only stars, planets, star clusters, galaxies, and the fundamental circles can be projected. Another difficulty is the manual control of the apparent motions of the planets. In any case, the facilities are not a place for entertainment; they are a place for pedagogic experiences.

Ten years ago, almost no astronomy was taught in French schools. A small group of teachers decided to begin extensive training of their colleagues. Thanks to their enormous efforts, different programs in astronomy are now included in both primary and secondary curricula. 


\section{Fundamental Aims of Planetariums}

\section{A. Discovery of Constellations}

Children living in cities are unable to observe the night sky and do not usually know the locations of the cardinal points. So, allowing for the ages of the children, it is essential to show them how to find these through the motion of the sun and the direction of Polaris. Next we begin a description of the main constellations and how to recognize them. At the very start of the session, they should be shown for the current season, so that the students can easily find them in the sky. The session could then develop into a discussion of the names and meanings of the zodiacal constellations.

Pointing out the relative brightness of stars provides the opportunity to tell students about stellar distances and about the nature and origin of starlight.

\section{B. Diurnal Movement}

A complete rotation of the Earth can take place in three to twelve minutes, depending on the planetarium mode. In either case, the speed is sufficient to illustrate the phenomenon. Most students have not noticed this nighttime movement of stars and associated it with the diurnal motion of the sun; however, we regard this observation as one of the most important to explain.

We place special emphasis on the locations of rising stars, the duration of their visibility, and on their culmination. It is possible to compare star trails near the celestial pole with those at the celestial equator, and in the southern hemisphere.

\section{Annual Solar Movement}

In most planetariums, annual solar movement along the ecliptic is made manually. Doing so is not especially easy or convenient, but the ecliptic and equatorial circles aid the demonstrations, and with secondary-school students, it is possible to explain the seasons and the variations in the night sky throughout the year. By changing the apparent latitude of the observer, the reason for the "polar night" and the "midnight sun" can easily be demonstrated.

\section{Planetary Movements}

As previously stated, the small commercial planetariums are not well equipped to exhibit the annual motion of planets among the stars. However, students are most interested in information about the nature of the planets, and it is a pleasure to encourage them to talk about it. At this moment in the session, the proximity of the planets to Earth compared with the stars can be pointed out, and their relative brightnesses and physical properties can be explained.

Venus is a good (and easily observed) example of the apparent motion of a planet around the sun and illustrates the phenomenon of the phases of illumination. 


\section{E. Lunar Motion}

The motion of the moon is a topic of particular interest to students. The planetarium helps present a clear and understandable explanation of lunar phases, and of the moon's rapid monthly motion among the stars. These phenomena, emphasized by the visible phases of the moon at night, are easily comprehensible even by 10-year olds.

\section{Secondary Aims of the Planetarium}

Planetariums give also an opportunity to describe stellar distributions in clusters, such as M44 (Praesepe), and $h$ and $\chi$ Persei.

Some galaxies can be shown too, such as M31 and the Magellanic Clouds. The location of the Milky Way should be clearly shown, although presenting it with a diffuser is only fair.

\section{How Transportable Planetariums are Utilized in Schools}

These facilities generally spend from a day to a week in each location. During this time, the whole student population or only selected classes may receive instruction.

Because of the limited space and ventilation, a standard session in the dome is no longer than 30-45 minutes. Still, transportable planetariums are perfect for children who visit a planetarium for the first time and whose curiosity is great. We allocate a short period in the dome and a longer period outside to answer the questions that inevitably arise from this curiosity.

The planetarium session is usually a part of a larger activity including exhibits, video or films, and a description of astronomical devices (celestial globes, sundials, astrolabes...). Whenever possible, we organize an outside session to observe the night sky.

We must point out that the person conducting this planetarium show can be a volunteer teacher with specific training, an amateur astronomer, or an enthusiastic astronomy student, but in all cases this activity is normally unpaid at the present time. We operate these programs with inflatable planetariums 25 to 30 weeks a year. They serve about 10,000 students a year.

\section{A Unique Experience in Nice}

At Valeri's College in Nice, children from 12 to 15 years of age decided to build their own planetarium in the schoolyard. Sixty square meters were allocated, and in 1985, a facility was completed that included a planetarium with a four-meter dome, an observatory, and an astronomical laboratory.

The students, who are very proud of their accomplishment, were greatly motivated to produce exhibits and models for display in the surrounding schools and general community. This project is a remarkable example of the student's enthusiasm for astronomy. 


\section{Concluding Remarks}

Most large cities in France do not have a planetarium. This lack is a source of frustration to teachers who are aware of the great interest that their students have in astronomy. The inflatable planetariums are an answer to this need.

Their number has been increasing in these last three years, but as no resources are yet available from official agencies, encouraging this activity is a most difficult problem and necessitates the use of highly motivated volunteers. In any case, we feel that increased planetarium use must develop in France and in many other countries in the context of a comprehensive plan for the better teaching of science.

\section{The Pre-College Planetarium}

\section{Brendan Curran \\ Bronx High School of Science, 75 West 205 St., Bronx, New York, New York, U.S.A.}

The Bronx High School of Science is a public school in the New York City school system. It is a specialized school whose students are selected on the basis of a competitive exam. Our planetarium projector was installed out his spring, so many of these suggested activities are still in development stage.

In the high school setting, the planetarium has a dual role as a teaching tool and as a motivational tool. The planetarium is used in the Astronomy and Astrophysics course, an advanced elective. This use permits a detailed study of the sky, which is otherwise difficult in New York City. Students who are familiar with thinking in two dimensions often have trouble making the transition to the three dimensions required in astronomy. The planetarium is an invaluable tool to develop students' spatial orientation. Further, the planetarium generates tremendous enthusiasm.

The planetarium will also find a place in the physics curriculum, maximizing its exposure to students since every student must take the physics course. Students will be given a planetarium experience when they study geocentric versus heliocentric models of the solar system and Kepler's laws of planetary motion. The planetarium will provide reinforcement, making the lessons memorable.

An additional benefit will be to generate interest in astronomy. The result will be a supply of students for the course as well as encouraging amateur interest.

We hope to develop additional programs, particularly some linking the humanities and astronomy. When English classes learn mythology, a visit to the planetarium would highlight the connection between culture and the sky. Planetarium observation would be appropriate for art-history classes. In social studies, a discussion of various calendars used by different human societies and their relation to celestial motion would provide enrichment.

We hope that these programs will maximize the use of the planetarium and increase students' awareness of astronomy. 